\section{RSP}

http://www.rsp.fsp.usp.br/
Revista de Saúde Pública

\title{
Violência em transporte público: uma abordagem baseada em análise espacial
}

\author{
Daiane Castro Bittencourt de Sousa', Cira Souza Pitombo", Samille Santos Rocha", Ana Rita \\ Salgueiro'"I', Juan Pedro Moreno Delgado' \\ ' Universidade Federal da Bahia. Escola Politécnica. Departamento de Engenharia de Transportes e Geodésia. \\ Salvador, BA, Brasil \\ " Universidade de São Paulo. Escola de Engenharia de São Carlos. Departamento de Engenharia de Transportes. \\ São Carlos, SP, Brasil \\ III Universidade Federal do Ceará. Centro de Ciências. Departamento de Geologia. Fortaleza, CE, Brasil
}

\section{RESUMO}

OBJETIVO: Realizar uma análise espacial da ocorrência de atos de violência (especificamente roubos) em transporte público, identificando as regiões de maior incidência, por meio da geoestatística, e possíveis causas com auxílio de análise multicritério em Sistema de Informação Geográfica.

MÉTODOS: A unidade de análise é a zona de tráfego da pesquisa Origem-Destino, realizada em Salvador, BA, Brasil, em 2013. Os roubos registrados pela Secretaria de Segurança Pública da Bahia, no mesmo ano, foram localizados e compatibilizados aos limites das zonas de tráfego e, posteriormente, associados aos respectivos centroides. Após determinação das regiões de maior probabilidade de ocorrências, foi feita uma análise geográfica de possíveis causas na região de maior potencial, considerando fatores analisados por meio de uma análise multicritério em ambiente de Sistema de Informação Geográfica.

RESULTADOS: A execução das duas etapas deste trabalho permitiu identificar áreas correspondentes à maior probabilidade de ocorrência de roubo em transporte público. Além disso, foram identificados três trechos viários (Estrada da Liberdade, Rua Pero Vaz e Avenida General San Martin) mais vulneráveis, localizados nessas áreas. Nesses trechos, os fatores que mais contribuem para o potencial de ocorrência de roubo em ônibus são: F1 - proximidade a locais que facilitam a fuga, F3 - grande movimentação de pessoas e F2 - ausência de policiamento, respectivamente.

CONCLUSÕES: Com o uso da Krigagem indicativa (estimação geoestatística), é possível a construção de uma superfície espacial de probabilidades de ocorrências, que pode ser uma ferramenta útil para implementação de políticas públicas. A análise multicritério no ambiente do Sistema de Informação Geográfica permitiu a compreensão dos fatores espaciais relacionados ao fenômeno em análise.

DESCRITORES: Roubo, estatística \& dados numéricos. Transportes. Análise Espacial. Sistemas de Informação Geográfica, utilização. Violência, prevenção \& controle. 


\section{INTRODUÇÃO}

Diariamente, no espaço viário urbano das grandes cidades brasileiras, motoristas, cobradores de ônibus e passageiros são expostos à poluição sonora e atmosférica, congestionamentos e acidentes de trânsito e no trabalho, bem como a atos de violência.

A violência no transporte público afeta um dos direitos do cidadão - a mobilidade -, além de estar efetivamente relacionada à saúde pública. A insegurança no interior dos ônibus (ocorrência de acidentes durante o deslocamento e atos de violência) compromete a qualidade do transporte nas cidades ${ }^{6}$.

A qualidade no transporte público passou a ser entendida, a partir dos anos 1990, no contexto dos parâmetros da qualidade pela visão do usuário, ou seja, deixou de ser analisada no campo operacional para contemplar as expectativas do usuário e necessidades dos cidadãos em relação ao transporte público. Assim, a qualidade, além de ser um parâmetro quantitativo, passa a ser considerado também qualitativo, pois envolve a satisfação do uso 4 .

A redução da qualidade do serviço de transporte público provoca maior procura pelo uso do automóvel e outros modos de transporte individuais. As consequências desse problema são facilmente percebidas pelo aumento de veículos nas vias, aumentando a ocorrência de congestionamentos e acidentes de trânsito e, consequentemente, elevação no tempo de viagem. O congestionamento é visto como um dos principais fatores que prejudicam a qualidade de vida das pessoas e eficiência do sistema de transporte ${ }^{3}$.

Dessa maneira, deve haver uma valorização do transporte público em detrimento do transporte individual motorizado, proporcionando qualidade e eficiência, de acordo com as necessidades de mobilidade da população. Isso envolve uma melhora na segurança relacionada ao sistema de transporte público, principalmente na redução de roubo em ônibus.

Na abordagem da violência no transporte coletivo por ônibus, a problemática dos roubos corresponde à dimensão da segurança pública, somados a outros problemas, como furtos, assédio e violência social e política. Estudos mostram que a diminuição no número de usuários de ônibus, principalmente nos horários de menor movimento, ocorre em virtude da alta incidência de roubos ${ }^{13}$.

Mendes $^{14}$ analisou as ocorrências de roubo em ônibus na cidade de Uberlândia, MG, entre os anos de 2005 e 2006, e identificou algumas características dos bairros que se destacaram com maior número de roubos, a saber: alta demanda de passageiros e localização periférica. Especificamente em Salvador, BA, Paes-Machado e Levenstein ${ }^{16}$ desenvolveram um estudo que aborda o impacto da criminalidade violenta nas condições de trabalho, saúde e segurança dos trabalhadores de transporte coletivo, utilizando um método qualitativo. Com a análise do perfil dos agressores envolvidos com o crime em transporte público por ônibus, os autores observaram que os mesmos geralmente são jovens, desempregados e sem antecedentes criminais.

Assunção e Medeiros ${ }^{2}$ testaram se fatores sociodemográficos e de condições de trabalho estavam associados à violência contra profissionais de ônibus de região metropolitana (três cidades da Região Metropolitana de Belo Horizonte, Brasil). Nesse estudo, a idade do trabalhador rodoviário mostrou-se inversamente associada à violência. Doenças crônicas, absenteísmo-doença e condições de trabalho também foram associados à violência² .

Estudo desenvolvido por Hart e Miethe ${ }^{8}$ analisou a violência em torno de pontos de ônibus e em outros nós do sistema na Austrália. Os autores se basearam em fundamentos da criminologia ambiental para o desenvolvimento da análise e identificaram que as paradas de ônibus são os locais de mais provável ocorrência de roubo quando comparadas a qualquer outro nó da rede.

Algumas cidades apresentam localizações de maior frequência de casos de violência no transporte público, sendo possível utilizar a análise espacial para estudos sobre o fenômeno. Acredita-se que o roubo em ônibus possui uma dimensão territorial e, 
geralmente, um padrão de distribuição espacial, o que possibilita a inserção de técnicas que considerem o atributo espacial, como o uso de geoestatística. Assim, a proposta é incorporar técnicas espaciais, aprimorando as análises com a utilização de um atributo adicional, a localização geográfica dos eventos. A partir de técnicas de geoestatística, é possível identificar a estrutura espacial dos dados e conhecer aspectos que não estariam disponíveis por métodos tradicionais. Considerando a técnica mencionada, é possível estimar probabilidades de ocorrência de roubo em transporte coletivo em coordenadas geográficas de valores conhecidos e desconhecidos.

A segunda ferramenta espacial utilizada neste trabalho para investigação dos fatores espaciais relacionados ao roubo em ônibus é a análise multicritério em Sistema de Informação Geográfica (SIG).É uma técnica que permite avaliar e agregar muitos critérios, que posteriormente são representados na análise como planos de dados geográficos ${ }^{6}$. Dessa forma, este artigo visa realizar uma análise espacial da ocorrência de roubos em transporte público por meio de uma abordagem geoestatística (Krigagem indicativa), além de análise multicritério em SIG.

\section{MÉTODOS}

Os principais materiais utilizados nesta pesquisa foram os dados e aplicativos auxiliares. Os dados de roubos em ônibus coletivos utilizados se referem às ocorrências registradas em 2013 pelo Sistema de Gerenciamento Estatístico (SGE), da Secretaria da Segurança Pública da Bahia (SSP-BA $)^{17}$. Esses dados foram disponibilizados pela Superintendência de Gestão Integrada da Ação Policial (SIAP), pertencente ao respectivo órgão.

O SGE possui uma base georreferenciada dos logradouros de Salvador. Portanto, todos os registros lançados nesse sistema possuem uma coordenada do centroide do logradouro onde aconteceu o crime. Especificamente em relação ao delito de roubo em ônibus em 2013, do total de 1.210 registros realizados no período, 969 apresentam coordenadas (Figura 1, A). Como a informação da coordenada do fato para quantificar os roubos por zonas de tráfego (ZT) era imprescindível, somente 969 registros foram considerados nesse estudo.

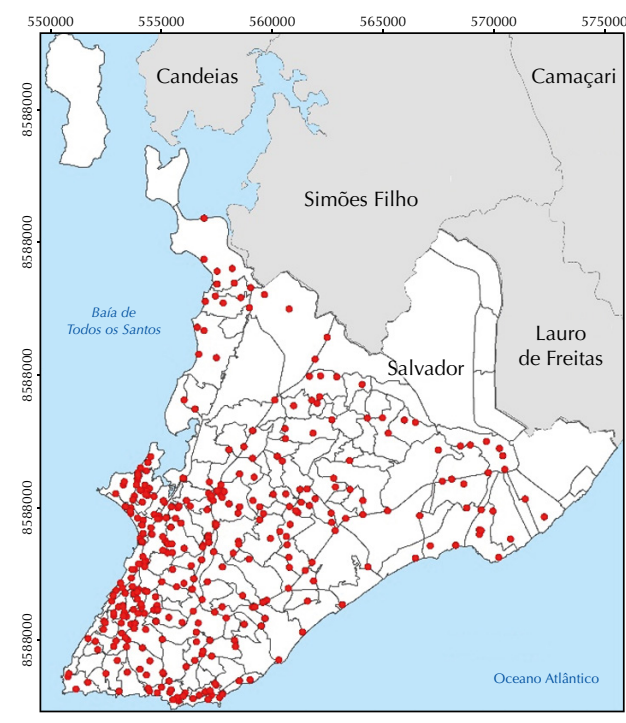

- Ocorrências de roubo em ônibus

$\square$ Região metropolitana

$\square$ Zonas de tráfego de Salvador

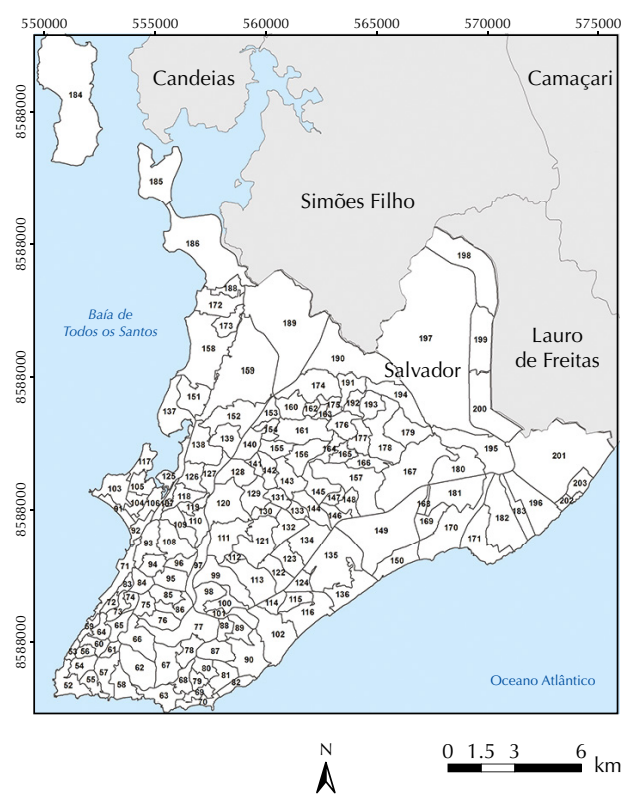

South American Datum (SAD) 69

Sistema Universal de Mercator (UTM)

Fontes: Secretaria da Segurança Pública da Bahia ${ }^{17}$ (2013). Secretaria de Infraestrutura da Bahia ${ }^{18}(2012)$.

Figura 1. Distribuição dos centroides dos logradouros com registro de roubo em ônibus (A) e zonas de tráfego (B). Salvador, BA, 2013. 
A unidade de análise deste estudo corresponde às 151 ZT de Salvador (Figura 1, B). Essas zonas, publicadas na última Pesquisa Origem-Destino ${ }^{18}$, tiveram seus limites definidos por meio da compatibilização com os limites de municípios e setores censitários do IBGE (2010) ${ }^{9}$.

As etapas que contemplam a análise geoestatística deste trabalho foram realizadas no programa ArcGis (Environmental Systems ResearchInstitute - ESRI), bem como a obtenção do total de roubos por ZT e os mapas resultantes da Krigagem indicativa. A avaliação multicritério foi realizada com auxílio do software IDRISI.

Este trabalho seguiu duas grandes etapas: (1) investigação geográfica do fenômeno Modelagem Geoestatística e (2) análise de possíveis causas: análise multicritério em SIG.

A primeira grande etapa corresponde à modelagem geoestatística. A geoestatística emergiu a partir da segunda metade do século XX. Posteriormente, o engenheiro francês Matheron $(1963)^{12}$, analisou os princípios dessa técnica e propôs o conceito de variáveis regionalizadas, e também introduziu a noção de variograma.

A partir de então, surgiram diversos estudos com aplicação de técnicas de geoestatística, principalmente na área de geologia ${ }^{5,11,19}$, hidrologia ${ }^{20}$, bem como nos estudos de petróleo e gás ${ }^{4}$. Mais recentemente, observa-se o uso dessa técnica na área de saúde pública, possibilitando estimação espacial de ocorrência de doenças, homicídios e violência urbana ${ }^{10,15,20}$.

A variável regionalizada refere-se ao fato de os valores observados não serem totalmente independentes, pois têm influência a partir de sua localização geográfica ${ }^{1}$. Logo, uma área com amostra de valor elevado apresenta maior probabilidade de os dados terem valores parecidos em sua proximidade.

Neste trabalho, a variável regionalizada seria número de ocorrência de roubos em ônibus, associado às coordenadas geográficas dos centroides das zonas de tráfego. Tal variável foi transformada em indicadora ( 0 ou 1 ), tendo em conta o valor de corte escolhido, para aplicação da Krigagem indicativa.

Assim, a estimação geoestatística de roubo em transporte público por ônibus foi realizada pela transformação de uma variável de contagem (quantidade de roubos) em variável dicotômica (zero $<$ valor de corte; $1 \geq$ valor de corte). $O$ valor de corte escolhido corresponde ao valor do $3^{\circ}$ quartil.

A modelagem geoestatística inicia-se a partir da obtenção dos variogramas experimentais e ajuste de variogramas teóricos. O variograma representa graficamente as informações da variável regionalizada. A função do variograma é determinada pela média das variâncias entre os pontos, tal como na seguinte equação:

$$
\gamma(h)=\frac{1}{2 N} \sum_{i=1}^{n(h)}[Z(x)-Z(x+h)]^{2}
$$

Sendo $N$ o número total de observações da amostra em cada distância $h$.

A partir dos conceitos matemáticos estabelecidos na equação, é feita a representação gráfica do variograma experimental. A etapa seguinte se refere ao ajuste do variograma teórico (modelos esférico e exponencial, por exemplo). Os parâmetros dos variogramas ajustados (efeito pepita, amplitude e patamar) são utilizados na etapa de estimação (Krigagem) como ponderadores para interpolação.

Assim, realizou-se o ajuste dos modelos teóricos e a obtenção de parâmetros importantes para estimação, como os valores de efeito pepita, amplitude e patamar. A Figura 2 ilustra o resultado do variograma teórico.

Para verificar a qualidade do modelo do variograma teórico, é imprescindível a etapa de validação cruzada, que é realizada a partir da comparação dos valores reais e estimados da variável regionalizada (VR) em análise. Também pode ser chamada de teste do ponto fictício, 


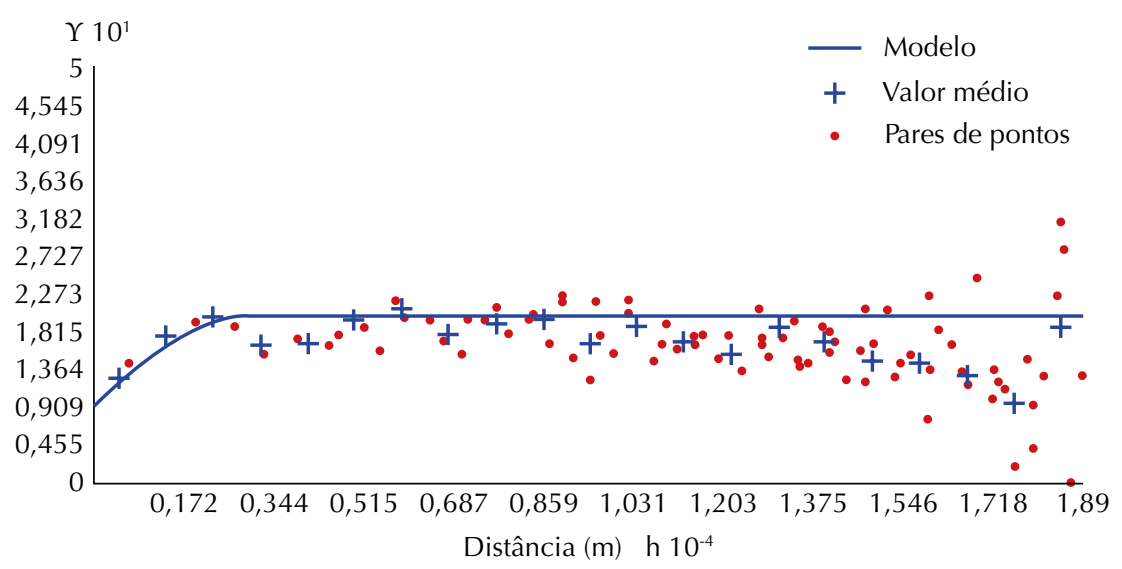

Figura 2. Variograma modelado para a variável binária roubo em ônibus: $3^{\circ}$ Quartil (Parâmetros do modelo: Tipo: Esférico. Direção principal: 37.1; Efeito Pepita: 0.091; Alcance: 2.600.00 m; Patamar: 0.187). Salvador, BA.

porque o ponto observado passa a ser ignorado, e estima-se um novo valor com base nos pesos das amostras vizinhas, estabelecidos pelo variograma teórico. Finalmente, haverá para cada ponto de mesma coordenada, o valor conhecido e o valor estimado, sendo possível mensurar a confiabilidade dos resultados usando medidas estatísticas de desempenho. No caso da Krigagem indicativa, o resultado da validação cruzada foi a probabilidade de ocorrência do fenômeno. Após discretizar as probabilidades estimadas e transformá-las em valores de 0 e 1, podem ser utilizados testes apropriados para variáveis qualitativas. Neste trabalho, foi analisado apenas o percentual de acertos da estimação por geoestatística.

A etapa seguinte à validação cruzada corresponde à estimação por Krigagem indicativa. A Krigagem permite-nos predizer valores em locais não amostrados, bem como fornecer uma estimativa do ponto com uma medida de acurácia associada. Um dos objetivos dessa medida é a minimização de erros relacionados à previsão de dados. Há, então, uma ponderação das amostras, o que geralmente reduz os erros de excesso, sendo essa a principal vantagem da krigagem ${ }^{1}$.

A Krigagem indicativa faz parte da categoria de estimadores não lineares da krigagem. Sua análise envolve desde a transformação dos dados originais, cálculo e modelagem do variograma, até a estimação da probabilidade de ocorrência do fenômeno analisado ${ }^{21}$. Esse tipo de krigagem possui a vantagem de não ser afetada por outliers, apresentando, portanto, variogramas mais facilmente ajustáveis quando comparados aos variogramas dos dados originais ${ }^{1}$. Os mapas interpolados, obtidos pela Krigagem são apresentados na seção de resultados.

A segunda grande etapa metodológica corresponde ao entendimento de possíveis causas de ocorrência dos roubos em transporte coletivo. Assim, foi realizada análise multicritério em SIG. Para realizar a avaliação multicritério, foram considerados os seguintes fatores identificados pelos especialistas como sendo os que mais influenciam na ocorrência de roubo em ônibus. F1 - proximidade a locais que facilitam a fuga; F2 - ausência de policiamento; F3 - maior frequência de ônibus; F4 - grande movimentação de pessoas; e F5 - proximidade a pontos de tráfico de drogas.

Foram consultados especialistas de segurança pública que trabalham diretamente com combate ao roubo em ônibus, e acadêmicos que desenvolveram pesquisas sobre o tema. Assim, a partir da aplicação de uma matriz de prioridade, os especialistas ponderaram cada fator em relação ao outro, em uma comparação par a par, respeitando as seguintes intensidades de importância - "muito mais importante" (10), "mais importante" (5), "igualmente importante" (1), "menos importante" (0,2), "muito menos importante" $(0,1)$ - a fim de determinar os pesos de cada fator.

As notas finais atribuídas a cada fator foram consideradas para determinação de seus respectivos pesos. Dessa forma, a combinação de todos os fatores na avaliação multicritério respeitou os pesos que resultaram da matriz de prioridade analisada pelos especialistas. 
Após isso, os planos de informação que representam cada fator foram mapeados a partir da coleta de informações em diferentes fontes e da realização do pré-processamento, quando necessário, utilizando preliminarmente o formato vetorial para a representação. Após o tratamento e atualização das informações utilizadas para mapear cada fator, houve a transformação do arquivo para o formato raster e a normalização e classificação fuzzy dos dados (variando de 0 a 1).

Com isso, todos os fatores mapeados foram integrados na análise multicritério pelo método de combinação linear ponderada em ambiente SIG. O produto dos pesos dos fatores pelos valores dos fatores determinou a variável chamada "potencial de roubos". O cálculo do "potencial de roubos", neste trabalho, foi realizado em três trechos viários pertencentes à região determinada como crítica na modelagem geoestatística.

Vale ressaltar queéuma abordagem sequencial eespacial para um caso específico de violência urbana. A primeira etapa - modelagem geoestatística - destina-seà determinação deáreas geograficamente potenciais à ocorrência de roubos em transporte coletivo. Já a etapa final - análise multicritério em SIG - corresponde ao entendimento dos fatores relacionados ao delito - roubo em ônibus.

\section{RESULTADOS}

A apresentação dos resultados é sumariada em duas etapas principais: (i) apresentação de mapa de valores estimados; e (ii) apresentação dos resultados da análise multicritério em SIG.

\section{Apresentação dos Mapas de Valores Estimados}

A validação cruzada faz o teste de qualidade do modelo dos variogramas ajustado ou teórico para valor de corte da variável "número de roubos a ônibus" equivalente ao $3^{\circ}$ quartil. Verificada a qualidade para os modelos variográficos (no caso deste trabalho, houve a acurácia de $75 \%$ de acertos), os parâmetros dos variogramas teóricos são utilizados na etapa seguinte da modelagem geoestatística - A Krigagem indicativa.

Assim, são gerados mapas de superfície ou mapas de valores estimados considerando uma malha de krigagem, valores da variável, localizações espaciais e parâmetros dos variogramas teóricos. O mapa de superfície, que estima a probabilidade da variável analisada, é apresentado na Figura 3. As áreas
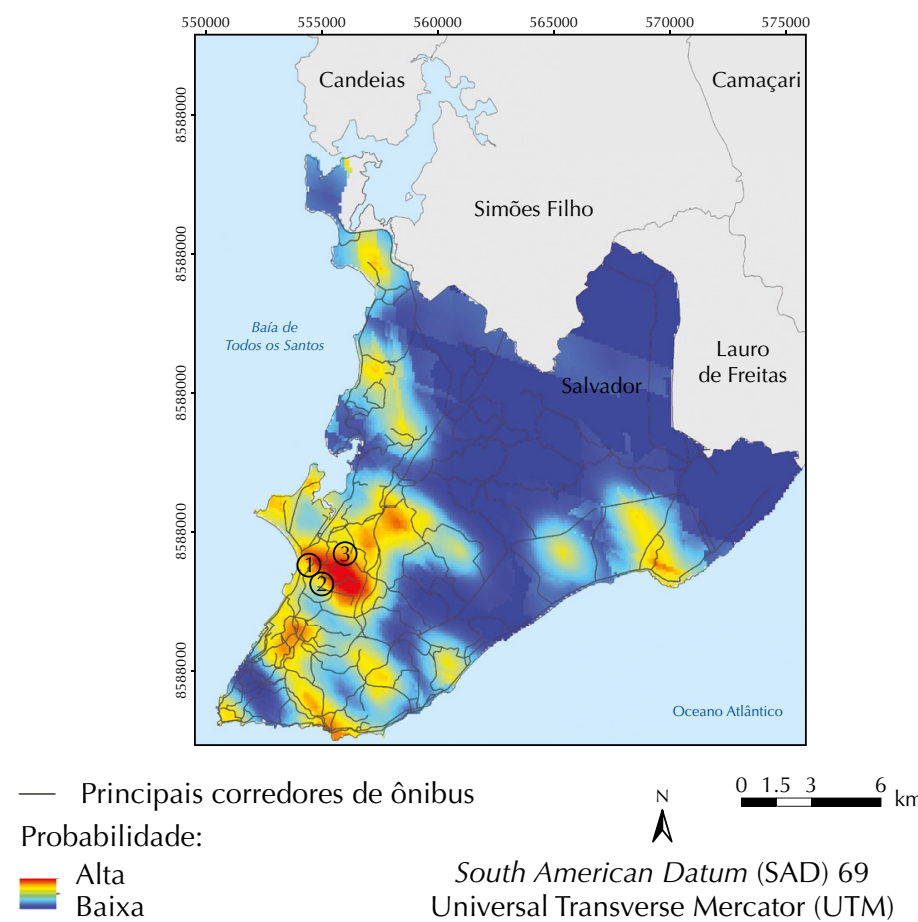

Fonte: Secretaria da Segurança Pública da Bahia ${ }^{17}$ (2013).

Figura 3. Mapa de probabilidade de ocorrências de roubo em transporte coletivo por ônibus. Salvador, BA, 2013. 
com tons mais próximos ao vermelho correspondem ao maior risco de ocorrer uma quantidade de roubos acima do valor de corte adotado, enquanto as áreas com tons em azul correspondem à menor probabilidade. Estão ainda sinalizados na mesma figura os trechos viários que fizeram parte da análise multicritério, localizados na região de mais probabilidade de ocorrências.

\section{Apresentação dos Resultados da Análise Multicritério em SIG}

As notas finais atribuídas, pelos cinco especialistas, a cada fator, são listadas em seguida e foram consideradas para determinação de seus respectivos pesos. Nota-se que o fator com maior peso correspondeu ao F2 - ausência de policiamento, com 0,32.

- F1 - proximidade a locais que facilitam a fuga ( peso = 0,26);

- F2 - ausência de policiamento (peso = 0,32);

- F3 - maior frequência de ônibus (peso = 0,17);

- F4 - grande movimentação de pessoas (peso = 0,13);

- F5 - proximidade a pontos de tráfico de drogas (peso = 0,12).

Após mapeamento dos valores correspondentes a cada fator no espaço urbano e cálculo do produto entre os seus respectivos pesos e valores atribuídos, procedeu-se ao cálculo da variável "potencial de roubos" em três trechos viários contidos na área mais crítica. Os trechos analisados são ilustrados na Figura 4: Trecho 1 - Estrada da Liberdade; Trecho 2 - Rua Pero Vaz; Trecho 3 - Avenida General San Martin.

Ao analisar detalhadamente o trecho crítico de ocorrência de roubo em ônibus na Estrada da Liberdade (Trecho 1), observa-se que os fatores que mais contribuem para o potencial de ocorrência de roubo em ônibus são: F1 - proximidade a locais que facilitam a fuga, F3 - grande movimentação de pessoas e F2 - ausência de policiamento, respectivamente (Figura 5, A).

Especificamente na Rua Pero Vaz (Trecho 2), o fator sobre proximidade a locais que facilitam a fuga (F1) apresentou contribuição máxima para determinação do potencial de ocorrência de roubo em ônibus, seguido dos fatores F3 - grande movimentação de pessoas e F2 - ausência de policiamento, respectivamente (Figura 5, B).

Na Avenida General San Martin (Trecho 3), o fator F1 - proximidade a locais que facilitam a fuga também apresenta contribuição máxima para o potencial de roubo em ônibus nesse trecho, seguido do fator F2 - ausência de policiamento. Os fatores que apresentaram menor

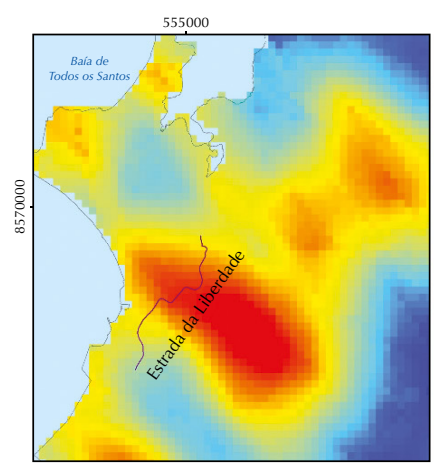

- Estrada da Liberdade

Probabilidade

- Alta

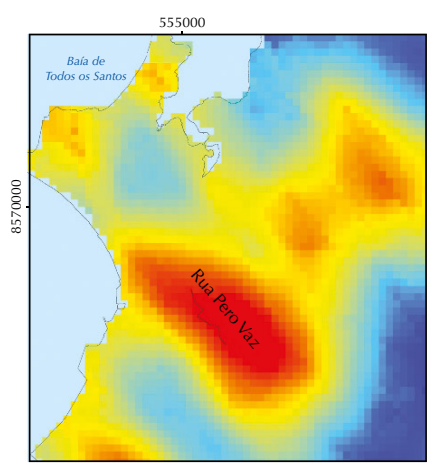

- Rua Pero Vaz

\section{$\AA^{N}$}

South American Datum (SAD) 69

Universal Transverse Mercator (UTM)

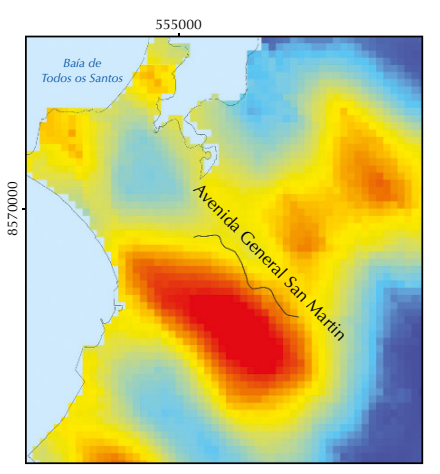

- Avenida General San Martin

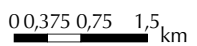

Figura 4. Mapa de probabilidade de ocorrências de roubo em transporte coletivo por ônibus. Salvador, BA, 2013. 

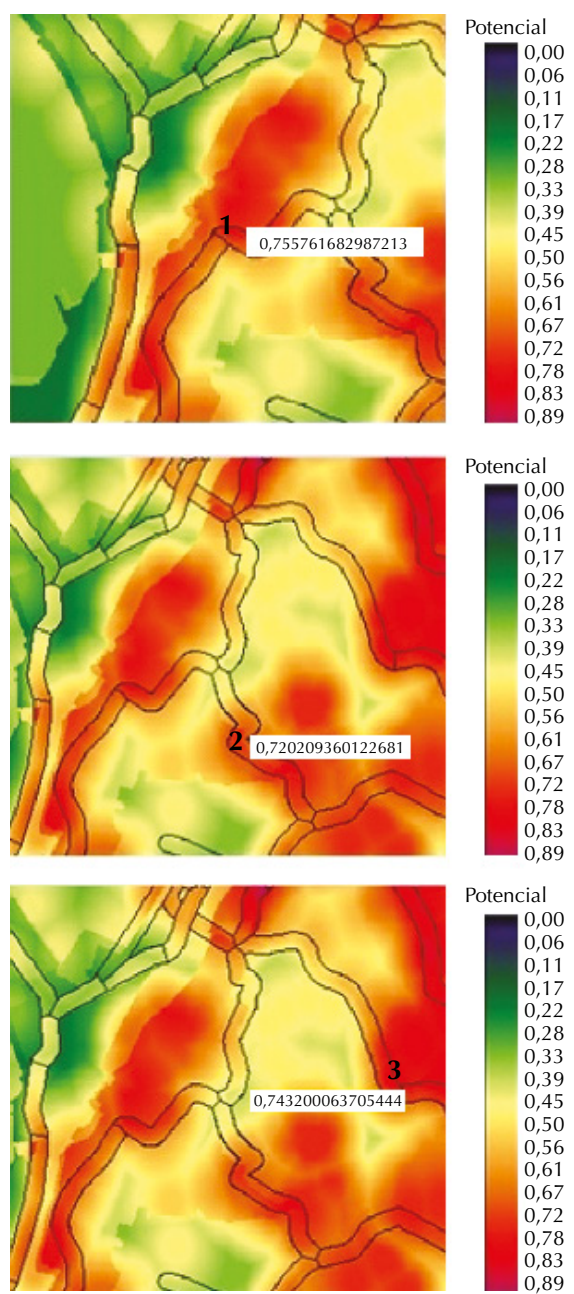

(A)

\begin{tabular}{ll}
\hline Atributo & Valor \\
\hline Fuga & 0,98 \\
\hline Ônibus & 0,25 \\
\hline Pessoas & 0,88 \\
\hline Policiamento & 0,87 \\
\hline Drogas & 0,51 \\
\hline
\end{tabular}

(B)

\begin{tabular}{lc}
\hline Atributo & Valor \\
\hline Fuga & 1 \\
\hline Ônibus & 0,08 \\
\hline Pessoas & 0,88 \\
\hline Policiamento & 0,87 \\
\hline Drogas & 0,41 \\
\hline
\end{tabular}

(C)

\begin{tabular}{lc}
\hline Atributo & Valor \\
\hline Fuga & 1 \\
\hline Ônibus & 0,3 \\
\hline Pessoas & 0,53 \\
\hline Policiamento & 0,94 \\
\hline Drogas & 0,49 \\
\hline
\end{tabular}

Figura 5. Análise do potencial de ocorrência de roubo em ônibus na Estrada da Liberdade (Trecho 1), na Rua Pero Vaz (Trecho 2) e na Avenida General San Martin (Trecho 3). Salvador, BA, Brasil.

contribuição foram: F3 - maior frequência de ônibus e F5 - proximidade a pontos de tráfico de drogas, conforme detalhe apresentado na Figura 5 (C).

\section{DISCUSSÃO}

A análise espacial através da Krigagem indicativa foi considerada eficaz na determinação das zonas de tráfego e regiões de maior probabilidade de ocorrências de roubo em ônibus. As zonas com maior ocorrência de roubos são detectadas nas análises obtidas pela Krigagem indicativa, como a região central, península e miolo de Salvador.

Uma vantagem da utilização da Krigagem indicativa em relação a outros tipos de krigagem na previsão de variáveis com comportamento pouco variável e com presença de outliers é a obtenção de variogramas facilmente modeláveis, o que amplia o poder preditivo a partir da relação espacial da variável analisada. Observa-se também que, com essa técnica, é possível obter dados em pontos que não foram amostrados, a partir de uma superfície contínua de dados que evidenciam áreas propensas à ocorrência de violência no transporte coletivo. Dessa forma, por meio dos procedimentos metodológicos seguidos, são obtidas manchas urbanas que indicam maior probabilidade de roubos a ônibus. A partir do valor de corte determinado neste estudo, foi possível identificar manchas associadas à probabilidade do número de ocorrências superiores ao valor do $3^{\circ}$ quartil para o ano de 2013.

Através de modelagem geoestatística, considerando apenas autocorrelação espacial da variável regionalizada, é possível estimar tais probabilidades inclusive em localizações de valores 
desconhecidos. As variâncias da variável objeto de estudo em relação a distâncias (variografia) conseguem detectar áreas de risco na região de estudo, com boa acurácia (75\% de acertos).

Uma análise complementar ou sequencial à modelagem geoestatística foi a análise multicritério em SIG, que realizamos neste trabalho com objetivo de detectar, considerando fatores espaciais, as principais causas na região de maior potencial de roubos. Observou-se que os fatores que mais contribuíram para determinação do alto potencial foram F1 - proximidade a locais que facilitam a fuga, F3 - grande movimentação de pessoas e F2 - ausência de policiamento.

A observação dessas tendências espaciais ocorre de maneira confirmatória, pela geoestatística. A análise posterior permite melhor investigação do fenômeno. Assim, o emprego desse procedimento metodológico pode auxiliar no processo de planejamento urbano e regional e implementações de políticas de segurança pública com objetivo de garantir o direito à mobilidade do cidadão.

\section{REFERÊNCIAS}

1. Andriotti JLS. Fundamentos de estatística e geoestatística. São Leopoldo: Unisinos; 2003.

2. Assunção AA, Medeiros AM. Violence against metropolitan bus drivers and fare collectors in Brazil. Rev Saude Publica. 2015;49:11. https://doi.org/10.1590/S0034-8910.2015049005380

3. Banister D. The sustainable mobility paradigm. Transp Policy. 2008;15(2):73-80. https://doi.org/10.1016/j.tranpol.2007.10.005

4. Barouch E, Kaufman GM. The interface between geostatistical modeling of oil and gas discovery and economics. J Int Assoc Math Geol. 1978;10(5):611-27. https://doi.org/10.1007/BF02461989

5. De Geoffroy J, Wignall TK. A statistical study of geological characteristics of porphyry-coppermolybdenum deposits in the Cordilleran Belt-Application to the rating of porphyry prospects. Econ Geol. 1972;67(5):656-68. https://doi.org/10.2113/gsecongeo.67.5.656

6. Eastman JR. IDRISI for Windows: introdução e exercícios tutoriais. Porto Alegre: Centro de Recursos IDRISI da UFRGS; 1998.

7. Ferraz ACP, Torres IGE. Transporte público urbano. São Paulo: RIMa; 2004.

8. Hart TC, Miethe TD. Street robbery and public bus stops: a case study of activity nodes and situational risk. Secur J. 2014;27(2):180-93. https://doi.org/10.1057/sj.2014.5

9. Instituto Brasileiro de Geografia e Estatística. Censo Demográfico Brasileiro 2010. Rio de Janeiro: IBGE; s.d. [citado 4 nov 2013]. Disponível em: https://downloads.ibge.gov.br/ downloads_estatisticas.htm

10. Jaime-Garcia R, Orum TV, Felix-Gastelum R, Trinidad-Correa R, VanEtten HD, Nelson MR. Spatial analysis of Phytophthora infestans genotypes and late blight severity on tomato and potato in the Del Fuerte Valley using geostatistics and geographic information systems. Phytopathology. 2001;91(12):1156-65. https://doi.org/10.1094/PHYTO.2001.91.12.1156

11. Klinge $U$. [On the possibilities for application of geostatistics in the evaluation of deposits, especially of uranium ores]. Erzmetall. 1971;24(5):220-6. German.

12. Matheron G. Principles of geostatistics. Econ Geol. 1963;58(8):1246-66. https://doi.org/10.2113/gsecongeo.58.8.1246

13. Medeiros FW. Assaltos no transporte coletivo urbano de Fortaleza [monografia]. Fortaleza: Universidade Federal do Ceará; 2008. Curso de Especialização em Gestão de Transportes Urbanos.

14. Mendes EO. Violência urbana: análise dos roubos a ônibus no SIT - 2005 e 2006 Uberlândia-MG. Uberlândia: Instituto de Geografia da Universidade Federal de Uberlândia; 2008.

15. Nicholson MC, Mather TN. Methods for evaluating Lyme disease risks using geographic information systems and geospatial analysis. J Med Entomol. 1996;33(5):711-20. https://doi.org/10.1093/jmedent/33.5.711

16. Paes-Machado E, Levenstein, C. Assaltantes a bordo: violência, insegurança e saúde no trabalho em transporte coletivo de Salvador, Bahia, Brasil. Cad Saude Publica. 2002;18(5):1215-27. https://doi.org/10.1590/S0102-311X2002000500014 
17. Secretaria da Segurança Pública da Bahia. Sistema de Gerenciamento Estatístico: centroides de ocorrências de roubo em ônibus. Salvador: SSP; 2013.

18. Secretaria de Infraestrutura da Bahia, Departamento de Infraestrutura de Transportes. Pesquisa de mobilidade na Região Metropolitana de Salvador: síntese dos resultados da pesquisa domiciliar. Salvador: SEINFRA; 2012 [citado 20 fev 2015]. Disponível em: http://www.seinfra.ba.gov.br/mobilidade2012/mobilidade.html

19. Sinclair AJ, Werner LJ. Geostatistical investigation of the Kutcho Creek chrysotile deposit, northern British Columbia. J Int Assoc Math Geol. 1978;10(3):273-88. https://doi.org/10.1007/BF02048491

20. Torellie L, Tomasi P. Interpolation and trend analysis: two geohydrological applications. J Int Assoc Math Geol. 1977;9(5):529-42. https://doi.org/10.1007/BF02100964

21. Yamamoto JK, Landim PMB. Geoestatística: conceitos e aplicações. São Paulo: Oficina de Textos; 2013.

Financiamento: Conselho Nacional de Desenvolvimento Científico e Tecnológico (CNPq - Processo 303645/2015-6 e Processo 142488/2014-3).

Contribuição dos Autores: Concepção e planejamento do estudo: DCBS,JPMD, CSP. Coleta, análise e interpretação dos dados: DCBS, SSR, ARS, CSP, JPMD. Elaboração ou revisão do manuscrito: DCBS, CSP, SSR. Aprovação da versão final: CSP, SSR. Responsabilidade pública pelo conteúdo do artigo: DCBS, CSP, JPMD.

Conflito de Interesses: Os autores declaram não haver conflito de interesses. 